\title{
Applying Genetic Algorithm to Optimization Second-order Bandpass MGMFB Filter
}

\author{
Maad Mohsin Mijwil* and Rana Ali Abttan \\ Computer Engineering Techniques Department, Baghdad College of Economics Sciences University, \\ Baghdad Province, 11001 Iraq
}

\begin{abstract}
In this paper, we have applied the genetic algorithm to the selection of the true values for RC (resistors/capacitors) as an essential role in the development of analogue active filters. The classic method of incorporating passive elements is a complex situation and can attend to errors. In order to reduce the frequency of errors and the human effort, evolutionary optimization methods are employed to select the RC values. In this study, Genetic algorithm (GA) is proposed to optimize the second-order active filter. It must find the values of the passive elements RC to get a filter configuration that reduces the sensitivities to variations as well as reduces design errors less than a defined height value, concerning certain specifications. The optimization problem which is one of the problems that must be solved by GA is a multi-objective optimization problem (MOOP). GA was

ARTICLE INFO

Article history:

Received: 24 March 2020

Accepted: 10 July 2020

Published: 21 October 2020

DOI: https://doi.org/10.47836/pjst.28.4.15 carried out taking into account two possible situations about the values that resistors and capacitors could adopt. The obtained experimental results show that GA can be used to obtain filter configurations that meet the specified standard.
\end{abstract}

E-mail addresses:

mr.maad.alnaimiy@baghdadcollege.edu.iq (Maad Mohsin Mijwil) rana.ali.abttan@baghdadcollege.edu.iq (Rana Ali Abttan)

*Corresponding author

Keywords: Genetic algorithm, optimization, passive elements, second-order bandpass filter, sensitivities 


\section{INTRODUCTION}

Active filters (AFs) are applied in high-frequency apps particularly in digital signal processing (DSP) circuits, communications, as well as health electronics (Soeiro et al., 2010; Shakoor et al., 2019). They can be executed in several ways, for example, using IC techniques, and requiring the presence of materials such as RC (Qazi \& Mustafa, 2016). The characteristics of these filters depend on the passive elements because the selection of these values is considered very significant due to their influence on these filters (Kaya \& Guler, 2018). There are various types of algorithms that are applied with AFs such as genetic algorithms (GAs), to find passive elements of RC (Benhala \& Bouattane, 2014). Theses algorithms are an artificial intelligence techniques inspired by the idea that the individual that survives is the one that is best adapted to the environment (Bhasin \& Bhatia, 2011). They are computational methods based on the theory of natural selection for the adaptive evolution of living things and have attracted attention as solutions to various optimization problems (Binitha \& Sathya, 2012). Genetic algorithms are used successfully for a variety of problems that do not allow an efficient solution through the application of conventional techniques (Cassar et al., 2017). Besides, these algorithms permit a population made up of a specific set of individuals to develop according to designated rules, to minimize/maximize fitness ( $f$ ) (Yan \& Wang, 2010; Yang et al., 2014).

Recently, the modern electronic design includes AFs in many applications, such as conditioning and signal handling at audio and intermediate frequencies (IF) as well as DSP tasks (Li et al., 2018). In contrast to digital filters, assets can obtain excellent performance with significantly lower power demands (Vural et al., 2012). The active filter implementation alternatives present many options (He \& Yin, 2018). The benefit of active filters is due essentially to integration ability and the extensive body of technical knowledge and they are very cheap, flexibility in gain and frequency adjustment, and no loading effect (Samadaei et al., 2011; Ibarra et al., 2011). Notwithstanding, the obtainable of active-filter ICs, most technicians and engineers are still resorting to RC active implementations consisting of operational amplifiers, RC (Corral, 2000; Zhang et al., 2020). Certainly, the prevalence of $\mathrm{RC}$ active filters has not declined since their bloom in the 1970s (Corral, 2000).

There are very few studies that apply the genetic algorithm to the second-order active filter. Starting with a study by Lovay et al. (2015), this study describes the application of the genetic algorithm to reduce sensitivities of second-order active filter called the SK (Sallen and Key) filter. The results show that configurations that meet established standards can be obtained. This study is considered closer to our study and its results have been compared to our results. In another study by Mostafa et al. (2018), eight components are applied as variables for improvement, as these components are compatible with the E96, E24, and E12 series by the genetic algorithm II (NSGA-II) with two analog filters which are a second order-filter and a fourth-order Butterworth with the operational amplifiers for testing 
purposes. The results of this study have been compared with seeker optimization algorithm, evolutionary algorithms, differential evolution, artificial bee colony, and harmony search.

Moreover, the collection of discrete elements in RC active filter is an aspect of great quality since compliance with the specifications will depend largely on the true choice from users (El Beqal et al., 2019; Alkhazraji \& Abttan, 2018). For reliable design, the values of the passive elements are selected from the E-series such as E12, E24, E48, \& E96. Each of these series limits the values that passive elements can assume. Also, the approximation of the values of the elements achieved by those available in the E-series impacts the quality of the filters but also limits the scope of optimization (Makama et al., 2018).

The objective of this paper is to apply GA to optimize active filters taking into account their sensitivities (Sens) and to verify the feasibility of the proposed method, a secondorder bandpass MGMFB (Multiple-Gain Multiple Feedback) filters is used. There are two possible situations about the values that resistors and capacitors can be used. For each situation, the algorithm must find the values of the elements that provides the established design requirements; and that reduces the sensitivities of the features of the filter concerning the values of the elements.

\section{MATERIALS AND METHODS}

\section{MGMFB filter}

MGMFB filter is widely applied as a bandpass filter because it allows an easy and reliable band-pass implementation, particularly below a quality factor $\left(Q_{p}\right)$ of 20 or so (Zumbahlen, 2012). Figure 1 shows the second-order bandpass MGMFB filter. The major benefit of this filter is that without any modifying in the maximum gain at the centre frequency we can modify the value of the cut-off frequency. This modifies in the cut-off frequency can be done by the R2.

Figure 1. The MGMFB filter

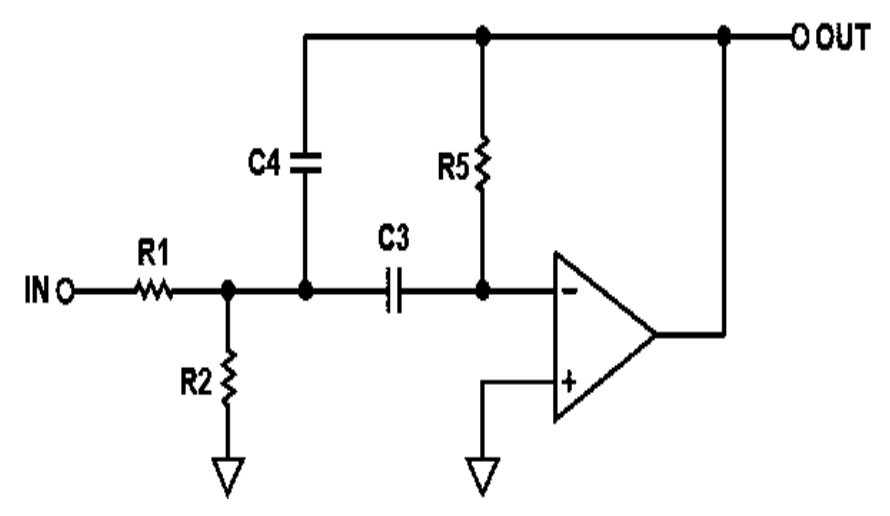


The circuit in Figure 1 produces an infinity MGMFB filter. Due to this circuit the $Q_{p}$ value increases maximum up to 20 .

The transfer function (TF) of a second-order bandpass filter, presented in the Laplace transform (LT) as in Equation 1.

$$
F(s)=\frac{-\frac{1}{R 1 C 3} s}{s^{2}+s\left(\frac{1}{R 5 C 3}+\frac{1}{R 5 C 4}\right)+\frac{1}{R 5 C 4}+\left(\frac{1}{R 1}+\frac{1}{R 2}\right)}
$$

This expression can be formulated based on the gain in the bandpass $(H)$, the pole frequency $\left(a_{p}=4 \pi f_{q}\right)$ and the $Q_{p}$, ordinarily referred to as filter specifications. The TF is presented in Equation 2.

$$
F(s)=\frac{1}{2 \pi} \sqrt{\frac{H a_{p}^{2}}{s^{2}+\left(\frac{a_{p}}{Q_{p}}\right) s+a_{p}^{2}}}
$$

For MGMFB filters, the values of, $a_{p}$, and $Q_{p}$ can be calculated from the values of the passive elements, according to Equation 3-5.

$$
\begin{aligned}
& H=\frac{R 5-C 3}{R 5 C 4+C 3} \\
& a_{p}=\sqrt{\frac{2 \pi}{R 2 * R 1 * C 4 * C 3}} \\
& Q_{p}=\sqrt{\frac{C 4}{C 3}}+\frac{\sqrt{R 1 R 5}}{0.5(R 1)}+\sqrt{\frac{R 5}{R 2}}+\frac{1}{2} \sqrt{\frac{R 2}{R 5}}
\end{aligned}
$$

Besides, MGMFB filters are less sensitive than other biquadratic filters. The less sensitive a filter to changes in its elements, the more stable the rest of its characteristics. Therefore, they are more likely to rest within its specifications, despite the presence of such variations.

Furthermore, if $f$ is a function of many variables, $f=\left(x_{1}, x_{2}, \ldots, x_{n}\right)$, then the sensitivity of $f$ concerning $x_{i}$ is defined by Equation 6:

$$
s_{x_{i}}^{f}=\frac{\% \text { change inf } f}{\% \text { change } \operatorname{in} x_{i}}=\frac{\partial f / f}{\partial x_{i} / x_{i}}
$$

A filter is considered to have low sensitivity when all its Sens use values lower than unity. For MGMFB bandpass filters, the sensitivities of $Q_{p}$ and $a_{p}$ concerning each of the passive elements are (Equation 7-12):

$$
\begin{aligned}
S_{R 1}^{a_{p}} & =0 \\
S_{R 2}^{a_{p}} & =S_{R 5}^{a_{p}}=S_{C 3}^{a_{p}}=S_{C 4}^{a_{p}}=\left|\left(\frac{1}{2}\right)\right|
\end{aligned}
$$




$$
\begin{aligned}
S_{C 3}^{Q_{p}} & =S_{C 4}^{Q_{p}}=\left|\left(\frac{1}{2}\right)\right| \\
S_{R 1}^{Q_{p}} & =Q_{p}\left(\frac{1}{0.5(R 1)} \sqrt{\frac{R 2 R 5 C 4}{C 3}}\right) \\
S_{R 5}^{Q_{p}} & =\frac{Q_{p}}{4}\left(\frac{1}{0.5(R 5)} \sqrt{\frac{R 2 R 3 C 4}{C 3}}-\sqrt{\frac{R 5 C 4}{R 2 C 3}}+\sqrt{\frac{R 2 C 4}{R 5 C 3}}\right) \\
S_{R 2}^{Q_{p}} & =\frac{Q_{p}}{4}\left(\frac{2}{R_{1}} \sqrt{\frac{R 2 R 5 C 4}{C 4}}-\sqrt{\frac{R 5 C 4}{R 2 C 4}}+\sqrt{\frac{R 5 C 3}{R 2 C 3}}\right)
\end{aligned}
$$

It is possible to estimate that in Equation 7-9 the sensitivities assume fixed values. In other words, the sensitivities specified in the remaining expressions indicate dependence on the values that the elements possess. For this purpose, the sensitivities expressed in Equation 10-12 must be recognized when choosing the values of both resistance and capacitor. In this paper, the specification chosen for the filter to be optimizing is (Equation 13-15):

$$
\begin{aligned}
& H_{f}=2 \\
& a_{p_{f}}=\frac{797 * 2 \pi * f}{2.5} \mathrm{rad} / \mathrm{s} \\
& Q_{p_{f}}=1.08 .
\end{aligned}
$$

\section{Optimization by the Genetic Algorithm}

This section describes the mechanism for using the GA to reduce the sensitivity of the filter, Figure. 2 presents a flow diagram of the GA.

Figure 2 presents the steps of the genetic algorithm, as follows:

$1^{\text {st }}$ step-Input samples: Insert all samples and parameters.

$2^{\text {nd }}$ step-Initialize population: This step includes the random generation of an initial population of individuals that are feasible solutions to the problem. In this study, every individual represents a filter configuration characterized by the values of RC. The GA describes every individual using a chromosome made up of 5 genes. Each gene represents the value of a resistor or a capacitor and is encoded using the entire encoding technique.

$3^{\text {rd }}$ step- Evaluation: In this step, every individual in the current population is estimated according to a predefined criterion, termed the fitness $(f)$. In this study, the MOOP in Equation (19) is converted into an optimization problem with a single objective. For this, the weighted sum method is applied, which uses an aggregation function to carry out the 


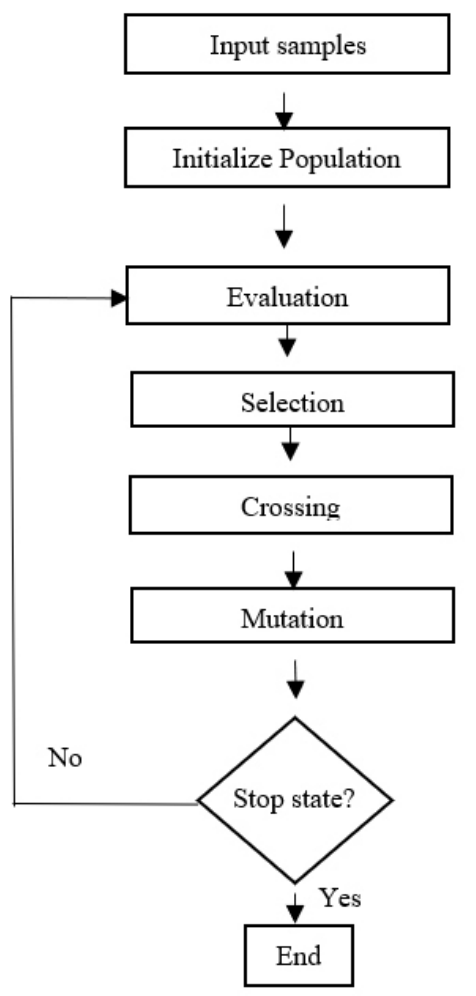

Figure 2. Flow diagram of GA steps

transformation. This is an easy way to get the best results. In this way, the $f$ that assigns a fitness value to every individual $(n)$, by Equation 16 :

$$
f(n)=\left(w_{1}\left|S_{R 1}^{Q_{p}}\right|+w_{2}\left|S_{R 2}^{Q_{p}}\right|+w_{3}\left|S_{R 5}^{Q_{p}}\right|\right)
$$

In Equation 16, $w_{i}$ denotes the weight assigned to each of the sensitivities total (Senstotal) that must be minimized. Because it is estimated that there is no preference between them, $w_{i}$ adopts the same value for the 3 Sens (Equation 17-18).

$$
\begin{aligned}
& f(n)=\frac{1}{3} \operatorname{Senstotal}(n) \\
& \operatorname{Senstotal}(n)=\left(\left|S_{R 1}^{Q_{p}}\right|+\left|S_{R 2}^{Q_{p}}\right|+\left|S_{R 5}^{Q_{p}}\right|\right)
\end{aligned}
$$

In other words, the GA punishes those individuals that do not comply with them in every iteration. So, they adopt a higher fitness value according to the degree of violation of each restriction. This procedure is applied to Equation 16. 
$4^{\text {th }}$ step-Selection: To produce a new population, the value of $f$ is essential in selected individuals. For this, the GA employs the spinning roulette method as a selection method, which supposes that the individual's choice to crossing depends on the value of their $f$.

$5^{\text {th }}$ step- Crossing: The elected individuals are subjected to the action of the crossing operator, which enables the replacement of genetic elements between them, making it reasonable to create new individuals to replace their parents, forming the population of the next generation.

$6^{\text {th }}$ step-Mutation: The population of individuals resulting from the $4^{\text {th }}$ step process is subjected to the mutation operator. It randomly affects an alteration in some of the genes on the chromosomes that make up the population, to avoid lack of diversity. For this purpose, the GA applies the consistent random mutation operator, which considers that each gene has the same probability of being mutated.

$6^{\text {th }}$ step- Stop state: The new generation passes through the process defined from the $2^{\text {nd }}$ step to the $5^{\text {th }}$ step process. The cycle is renewed till the stop state is fulfilled, including reaching a maximum number of generations.

Now, the GA find the passive elements values of the MGMFB (3 resistors \& 2 capacitors) for which the sensitivities that rely on the values of the elements are minimum, and the errors in $H, a_{p}$ and $Q_{p}$ are less than a maximum error specified. The MOOP may be described by the Equation 19 and it is a possible filter configuration determined by the resistor and capacitor values.

$$
\min f(n)=S_{R 1}^{Q_{p}}(n), S_{R 2}^{Q_{p}}(n), S_{R 5}^{Q_{p}}(n)
$$

Subject to: $\operatorname{Error}_{H}(n) \leq E_{\text {max }}$

$$
\begin{aligned}
& \operatorname{Error}_{a_{p}}(n) \leq E_{\text {max }} \\
& \operatorname{Error}_{Q_{p}}(n) \leq E_{\text {max }}
\end{aligned}
$$

Where:

$S_{R 1}^{Q_{p}}, S_{R 2}^{Q_{p}}, S_{R 5}^{Q_{p}}$ represents sensitivities that must be minimized, Error ${ }_{H}$, Error $_{a_{p}}$ and Error $_{Q_{p}}$ represent the errors in $H, a_{p}$ and $Q_{p}$, respectively and are calculated according to the Equation 20-22. Finally, $E_{\max }$ is described as the maximum common design error in the filter elements.

$$
\operatorname{Error}_{H}(n)=\left|\frac{H(n)-H_{f}}{H_{f}}\right|
$$




$$
\begin{aligned}
& \operatorname{Error}_{a_{p}}\left(n=\left|\frac{a_{p}(n)-a_{p_{f}}}{a_{p_{f}}}\right|\right) \\
& \operatorname{Error}_{Q_{p}}(n)=\left|\frac{Q_{p}(n)-Q_{p_{f}}}{a_{p_{f}}}\right|
\end{aligned}
$$

\section{RESULTS AND DISCUSSION}

The GA is aimed at optimization. The active filters are evaluated in this paper by taking into account the sensitivities and design errors obtained. For this, two possible situations are proposed to the values that the elements can adopt from the optimization process by GA. In situation 1, the capacitors and resistors can assume values according to the E24 and E96, respectively, considering that Emax $=5.00 \mathrm{E}-03$ (0.5\%). Moreover, situation 2, uses the E12 series for capacitor values and E24 series for resistance values, using the value 2.50E-02 (2.5\%) for Emax. In both situations the range of defined values is $10^{3}-10^{6} \Omega \&$ $10^{-9}-10^{-6} \mathrm{~F}$, respectively. The values of these ranges are assumed to have adverse impacts due to parasitic capabilities or very large modern signals. Therefore, the total search space amounts to $1.10 \mathrm{E} 09$ in situation 1 as well as 2.22E06 alternatives in situation 2 .

Table 1 shows the Senstotal values optimized by the GA. In situation 1, the GA obtains an appropriate solution for population sizes from 20 to 70 individuals and 3 crossover probabilities estimated. Senstotal reaches the lowest value with a population of 60 individuals and crossover probability 0.9. In situation 2, the population sizes from 10 to 70 , and 3 crossover probabilities are chosen. Senstotal reaches the lowest value with a population of 50 individuals and crossover probability 0.9 in situation 2 .

Table 1

Senstotal values obtained for different GA parameter values

\begin{tabular}{llllllll}
\hline & \multicolumn{3}{c}{ Situation 1 } & \multicolumn{5}{c}{ Situation 2 } \\
\hline Population & \multicolumn{3}{c}{ Crossover Probability } & Population & \multicolumn{3}{c}{ Crossover Probability } \\
\hline & 0.7 & 0.8 & 0.9 & & 0.7 & 0.8 & 0.9 \\
\hline 20 & 0.8544 & 0.6790 & 0.7237 & 10 & 0.8076 & 0.6703 & 0.8329 \\
30 & 0.6511 & 0.6725 & 0.9015 & 20 & 0.6852 & 1.1777 & 0.6541 \\
40 & 0.8647 & 0.7327 & 0.6516 & 30 & 0.8740 & 0.6440 & 0.6792 \\
50 & 1.2766 & 1.0110 & 1.0743 & 40 & 0.6690 & 0.8340 & 0.6542 \\
60 & 0.6725 & 0.6725 & 0.6430 & 50 & 0.6540 & 0.6540 & 0.6203 \\
70 & 0.6632 & 0.8329 & 1.0215 & 60 & 0.6540 & 0.6540 & 1.2046 \\
& & & & 70 & 0.6540 & 0.6540 & 0.6518 \\
\hline
\end{tabular}


Table 2 presents the results of the elements, their errors and the sensitivities achieved by the GA. In each situation, the described values correspond to the solution obtained by the genetic algorithm that has the lowest Senstotal value (Table 1). Besides, sensitivities reach similar values in both situations, with the exception of $S_{R_{S}}^{Q_{p}}$, where the result obtained from situation 1 is less than from the result obtained from situation 2. Nevertheless, Senstotal uses very similar values for the situations proposed. On the other hand, the specifications, (1) and (2) are applied to achieve the TF of a filter termed a NF (nominal filter). This filter does not consider into account the sensitivities, because it is not obtained using the values of its elements. But its frequency response (FR) is applied to graphically observe compliance with limitations by the solutions obtained by the GA. The FR of the NF and the response of the filter configuration are obtained by the algorithm in all situation.

Table 2

The result achieved by GA for each proposed situation

\begin{tabular}{lll}
\hline Parameter & Situation 1 & Situation 2 \\
\hline$R 1$ & $16600 \Omega$ & $10900 \Omega$ \\
$R 2$ & $36400 \Omega$ & $21000 \Omega$ \\
$R 5$ & $11000 \Omega$ & $6100 \Omega$ \\
$C 3$ & $1.21 \mathrm{E}-08 \mathrm{~F}$ & $0.40 \mathrm{E}-08 \mathrm{~F}$ \\
$C 4$ & $1.01 \mathrm{E}-09 \mathrm{~F}$ & $1.21 \mathrm{E}-09 \mathrm{~F}$ \\
$H$ & 2.0042 & 1 \\
$Q \mathrm{p}$ & 0.7066 & 0.7151 \\
$a_{p}$ & 5202.2015 & 4241.1778 \\
Error & $0.1622 \%$ & 0 \\
Error $Q_{p}$ & $0.0472 \%$ & $1.0083 \%$ \\
Error & $0.1916 \%$ & $1.3820 \%$ \\
$\left|S_{R 1}^{Q_{p}}\right|$ & 0.2641 & 0.2529 \\
& & \\
$\left|S_{R 2}^{Q_{p}}\right|$ & 0.2652 & 0.2564 \\
$\left|S_{R 5}^{Q_{p}}\right|$ & & \\
Senstotal & 0.0002 & 0.0011 \\
\hline
\end{tabular}


The GA implements a stochastic process, the results of this process may be changed according to the statistical distribution of the initial population. To observe how they can be influenced by the establishment of the initial population, 70 runs are made for each situation, changing the speed in the random generation of the initial population of each run. In both situations, it is possible to note that the three sensitivities always use values lower than 1. Also, $S_{R 1}^{Q_{p}}$ reaches the highest values and $S_{R 2}^{Q_{p}}$ has the lowest values, while $S_{R 5}^{Q_{p}}$ is the sensitivity with the lowest variations.

On the other hand, to complement the analysis, characterization of Senstotal and the errors received by the algorithm in the 70 runs performed are presented in Table 3 . In it, the minimum, maximum and median values are displayed for each situation. This measure of central tendency is adopted because the data presents a non-normal distribution. In the same table, it is possible to appreciate that Senstotal varies in a slightly greater range in situation 2, concerning situation 1 , presenting similar minimum values and a lower median in situation 2. As for errors, in both situations, the maximum error is less than $E_{\max }$ and minimum errors, in situation 1 the lowest value corresponds to Error ${ }_{a p}$ and the highest one to Error $_{H}$, while in situation 2, Error $_{H}$ reaches zero and the remaining two errors adopt similar values. For the median, the highest value corresponds to Error $_{G}$ in both situation, while the lowest value is presented for Error ${ }_{a p}$ in situation 1 and for Error ${ }_{Q p}$ in situation 2.

Table 3

Senstotal characterization and the errors obtained by the GA

\begin{tabular}{lllllll}
\hline & \multicolumn{3}{c}{ Situation 1 } & \multicolumn{3}{c}{ Situation 2 } \\
\cline { 2 - 7 } & Minimum & Maximum & Median & Minimum & Maximum & Median \\
\hline Senstotal & 0.6513 & 0.9322 & 0.7520 & 0.6521 & 2.4117 & 0.8710 \\
ErrorH $(\%)$ & 0.1682 & 0.3742 & 0.2409 & 0 & 1.2332 & 0.9223 \\
Error $_{a p}(\%)$ & 0.1269 & 0.4626 & 0.3721 & 0 & 2.4760 & 1.3539 \\
Error $_{Q p}(\%)$ & 0.0219 & 0.4126 & 0.2579 & 0.2232 & 3.3228 & 2.1731 \\
$\begin{array}{l}\text { Execution } \\
\text { times }\end{array}$ & 43,8345 seconds & & 51,7782 second & \\
$\begin{array}{l}\text { Estimated total } \\
\text { time }\end{array}$ & 472,100 seconds & & \multicolumn{3}{c}{2,330 seconds } \\
\hline
\end{tabular}

The GA gets an appropriate solution using considerably lower execution times than the exhaustive search method. Table 4 presents a comparison between the current results of this study and the previous study regarding the sensitivities (Sens) value of RC in both situations. 
Table 4

Comparison between current study and previous study

\begin{tabular}{lllllll}
\hline & \multicolumn{3}{c}{ Current study } & \multicolumn{3}{c}{ Previous study (Lovay et al., 2015) } \\
\hline Situation 1 & Minimum & Maximum & Median & Minimum & Maximum & Median \\
\hline Senstotal & 0.6513 & 0.9322 & 0.7520 & 1.994 & 2.222 & 1.964 \\
Situation 2 & Minimum & Maximum & Median & Minimum & Maximum & Median \\
\hline Senstotal & 0.6521 & 2.4117 & 0.8710 & 1.964 & 2.499 & 2.059 \\
\hline
\end{tabular}

The above table shows the difference between these two studies despite the difference in the type of filter used, but the genetic algorithm gave better results in the current study in terms of reducing sensitivities of RC values.

\section{CONCLUSIONS}

Within this work, a genetic algorithm to optimize the second-order bandpass MGMFB filter has been applied. For the values that RC can use, two possible situations are suggested. In each situation, the algorithm must find the values of the passive elements of the filter in order to have the configuration as robust as possible to the variations in them, by reducing the sensitivity. In addition, it should also be borne in mind that design errors relating to certain specifications must be less than the maximum value. Finally, the simulation outcomes indicate that the genetic algorithm can obtain filter configurations matching the defined requirements. Moreover, the outcomes can provide several design alternatives because of their stochastic nature.

\section{ACKNOWLEDGEMENT}

We thank the anonymous editors and reviewers for their useful suggestions to develop this manuscript.

\section{REFERENCES}

Alkhazraji, A. A., \& Abttan, R. A. (2018). Multiple fault detection and classification in power system. Advances in Natural and Applied Sciences, 12(5), 1-13.

Benhala, B., \& Bouattane, O. (2014). GA and ACO techniques for the analog circuits design optimization. Journal of Theoretical and Applied Information Technology, 64(2), 413-419.

Bhasin, H., \& Bhatia, S. (2011). Application of genetic algorithms in machine learning. International Journal of Computer Science and Information Technologies, 2(5), 2412-2415.

Binitha, S., \& Sathya, S. S. (2012). A survey of bio inspired optimization algorithms. International Journal of Soft Computing and Engineering (IJSCE), 2(2),137-151. 
Cassar, I. R., Titus, N. D., \& Grill, W. M. (2017). An improved genetic algorithm for designing optimal temporal patterns of neural stimulation. Journal of Neural Engineering, 14(6), 1-32.

Corral, C. (2000). Designing RC active filters with standard-component values. EDN, 45(24), 141-149.

El Beqal, A., Benhala, B., Garbay, A., Kotti, M., Fakhfakh, M., \& Zorkani, I. (2019, April 18-19). Synthesis of a current mode second order band pass filter using the genetic algorithm. In Proceedings of 5th International Conference on Optimization and Applications (ICOA) ( pp. 1-9). Kenitra, Morocco.

He, J., \& Yin, J. (2018, July 28-30). A practical evolution model for filter automatic design. In Proceedings of International Conference on Natural Computation, Fuzzy Systems and Knowledge Discovery (ICNCFSKD) (pp. 1-8). Huangshan, China.

Ibarra, F. S., Claros, M. C., Espinosa, R. M., Levy, E. O., \& Betancourt, L. P. (2011). Design of 2nd order low-pass active filters by preserving the physical meaning of design variables. Revista Mexicana de Física E, 57(1),1-10.

Kaya, T., \& Guler, H. (2018). A hybrid genetic algorithm for analog active filter component selection. AEU International Journal of Electronics and Communications, 86, 1-7.

Li, S., Zou, W., \& Hu, J. A. (2018). Novel evolutionary algorithm for designing robust analog filters. Algorithms Journal, 11(3),1-22.

Lovay, M., Peretti, G., \& Romero, E. (2015, July 30-31). Application of genetic algorithms in the design of robust active filters. In Proceedings of International Conference for Argentine School of MicroNanoelectronics, Technology and Applications (EAMTA) (pp. 01-06). Villa Maria, Argentina.

Makama, A., Binns, R., \& Basha, M. (2018, July 14-16). Application of an infinite gain multiple feedback filter in VLC link range extension. In Proceedings of IEEE 2nd International Conference on Circuits, System and Simulation (ICCSS) (pp. 1-7). Guangzhou, China.

Mostafa S. S., Horta N., Garcíac A. G. R., \& Dias F. M. (2018). Analog active filter design using a multi objective genetic algorithm. AEÜ-International Journal of Electronics and Communications, 93(1), 93-94.

Qazi, S. H., \& Mustafa, M. W. (2016). Review on active filters and its performance with grid connected fixed and variable speed wind turbine generator. Renewable and Sustainable Energy Reviews, 57(12), 420-438.

Samadaei, E., Lesan, S., \& Cherati, S. M. (2011, April 18-19). A new schematic for hybrid active power filter controller. In Proceedings of the 2011 IEEE Applied Power Electronics Colloquium (IAPEC) Malaysia (pp. 143-148). Johor Bahru, Malaysia.

Shakoor, A., Abbas, S., \& Abbas, Z. (2019). Optimization of design parameters of low pass filter using genetic algorithm. UW Journal of Science and Technology, 3(1), 55-60.

Soeiro, T., Biela, J., Linnér, J., Ranstad, P., \& Kolar, J. W. (2010, November 7-10). Line power quality improvement for ESP systems using multi-pulse and active filter concepts. In Proceedings of International Conference on IEEE Industrial Electronics Society (IECON 2010) (pp. 1-6). Glendale, USA.

Vural, R. A., Yıldırım, T., Kadioglu, T., \& Basargan, A. (2012). Performance evaluation of evolutionary algorithms for optimal filter design. IEEE Transactions on Evolutionary Computation, 16(1), 135-147. 
Yan, X., \& Wang, X. (2010). Fitness function of genetic algorithm in structural constraint optimization. In Proceedings of International Conference on Advances in Swarm Intelligence (ICSI 2010) (pp. 432-483). Berlin, Germany: Springer.

Yang, M. D., Yang, Y. F., Su, T.C., \& Huang, K. S. (2014). An efficient fitness function in genetic algorithm classifier for land use recognition on satellite images. The Scientific World Journal, 2014(264512), 1-12.

Zhang, C., Shang, L., Wang, Y., \& Tang, L. (2020). A CMOS programmable fourth-order butterworth activeRC low-pass filter. Electronics Journal-MDPI, 9(2), 1-9.

Zumbahlen, H. (2012). Multiple feedback band-pass design example. Massachusetts, USA: Analog Devices, Inc. 
